\title{
Comparative Study on Biodecolorization Capabilities of Indigenous Strains to Azo Dyes
}

\author{
Chin-Tang Chen ${ }^{1}$, Chia-Chyi Wu ${ }^{2}$, Bor-Yann Chen ${ }^{1}$, Chung-Chuan Hsueh ${ }^{1,}$, Mao-Lin Hsu ${ }^{1}$ \\ ${ }^{1}$ Department of Chemical and Materials Engineering, National Ilan University, Ilan, Taiwan \\ ${ }^{2}$ Department of Horticulture, National Ilan University, Ilan, Taiwan
}

\section{Email address:}

chen9923045@gmail.com (Chin-Tang Chen), angwu@niu.edu.tw (Chia-Chyi Wu), boryannchen@yahoo.com.tw (Bor-Yann Chen), cchsueh88@gmail.com (Chung-Chuan Hsueh)

${ }^{*}$ Corresponding author

\section{To cite this article:}

Chin-Tang Chen, Chia-Chyi Wu, Bor-Yann Chen, Chung-Chuan Hsueh, Mao-Lin Hsu. Comparative Study on Biodecolorization Capabilities of Indigenous Strains to Azo Dyes. Science Discovery. Vol. 4, No. 2, 2016, pp. 109-115. doi: 10.11648/j.sd.20160402.19

Received: February 27, 2016; Accepted: May 4, 2016; Published: May 18, 2016

\begin{abstract}
This study is to inspect the biodecolorization capabilities of indigenous strains to azo dyes (RR198, RR141, RBk5, RBu160, RBu171, DY86, RY84 and RG19). Shewanella sp. WLP72 isolated under selection pressure of decolorization of RBu160 upon gut microbial consortia of Babylonia areolate showed the optimal color removal capability. Due to this, WLP 72 had the highest capability to decolorize RBu160. Apparently, conditions of isolation of model dye(s) would affect dye-decolorizing capabilities of strains to be expressed. Moreover, the chemical structure effect of azo dyes would have a significant impact on decolorization efficiency as indicated through specific decolorization rate (SDR) of azo dyes by WLP72. The presence of electron-withdrawing groups (i.e., $-\mathrm{SO}_{3}{ }^{-}$) as the substituents at ortho- or para to azo bond would enhance decolorization efficiency. However, since the ortho substituent caused steric hindrance near azo linkage(s), azo dyes with para substituent could be more favorable than those with ortho substituent for decolorization. This study suggested that both the electronic characteristics of substituents in azo dyes and the positions of substituents related to azo bond on the aromatic ring all significantly affected the efficiency of biodecolorization of azo dyes.
\end{abstract}

Keywords: Azo Dyes, Species Isolation, Biodecolorizaiton, Chemical Structure Effect

\section{比較本土脫色菌對多種偶氮染料脫色能力之研究}

\author{
陳錦堂 ${ }^{1}$, 䣕家琪 ${ }^{2}$, 陳博彥 ${ }^{1}$, 薛仲娟 ${ }^{1 *}$, 徐茂霖 ${ }^{1}$ \\ 1化學工程與材料工程學系, 國立宜蘭大學, 宜蘭, 台灣 \\ ²園㙯學系, 國立宜蘭大學, 宜蘭, 台灣 \\ 邮箱 \\ chen9923045@gmai1.com（陳錦堂），angwu@niu. edu. tw（䣕家琪），boryannchen@yahoo.com. tw（陳博彥）, \\ cchsueh88@gmail. com（薛仲娟）
}

中文摘要: 本研究針對以本土脫色菌對偶氮染料脫色之能力進行評估分析。其中以RBu160染料篩選取自鳳螺之腔腸中 之Shewane11a sp. WLP72對多種偶氮染料(RR198、RR141、RBk5、RBu160、RBu171、DY86、RY84、RG19)均有不錯脫色 能力, 尤其對RBu160之脫色效率更為優異, 故推測篩選條件會對之後進行染料脫色有顯著影響。另外, 將WLP72做偶氮 染料之比脫色速率 (SDR) 進行評比, 探討偶氮染料化學結構效應對生物脫色的影響。由結果發現染料結構對生物脫色有 著重大影響, 若在偶氮隻鍵之鄰位或對位處具有較高陰電性的官能基 (即拉電子基) (例如: 磺酸基- $\mathrm{SO}_{3}^{-}$之類), 則均因為 
會降低偶氮鍵的電子雲密度, 以致於有利於偶氮染料之生物還原脫色反應。再者, 若此類高陰電性的官能基 (即拉電子 基) 在偶氮雙鍵的對位處，因為立體阻礙效應低於在鄰位處，更有利於脫色反應。

关键词：偶氮染料，菌種節選，生物脫色，化學結構

\section{1. 引言}

染整工業廢水由於色度高, 生物分解性低, 易造成環 境上的污染，形成環保單位必然之稽查重點汗染物。其中 偶氮染料更占具一般應用染料 $60 \%$ 以上，而且偶氮染料具 有 $(-\mathrm{N}=\mathrm{N}-)$ 之結構特徵, 在環境中更穩定。更由於染料易 合成。且成本低穩定且顔色多樣化, 常應用於紡織加工、 化妝品、食品、皮革和塑料等行業 [1]。而約 10-15\%在染 色過程中所用染料會不結合在纖維上 [2], 因此, 染料自 處理系統中被釋放導致環境污染。是以所排放出來的染料 廢水, 不僅汗染了河川環境 部份相關染料物質亦具毒性, 故對生態造成莫大之衝擊。儘管有物理、化學方法可有效 處理, 但由於考量物理、化學處理經濟可行性及二次汗染, 因此以環境較友善的微生物處理為宜 [3]。Khalid et al. , (2008) [4]提出, 偶氮染料雖然由厭氧條件下進行降 解, 但所降解之中間物若要再進一步降解 (如: 去甲基化, 去磺酸化、還裂解), 則需要在好氧條件下進行。Fernando et al. , (2014) [5]亦提出兩階段式降解法, 先將染料在厭
氧之微生物燃料電池中進行染料降解, 後再進行第二階段 好氧降解, 將有毒中間物再進一步生物轉化成無毒或毒性 較低之小分子。

\section{2. 材料與方法}

\section{1. 化學品}

為了解生物脫色之難易比較, 在依待測染料區分如下: 其中使用 8 種染料可分為二大類別 (採購於台灣永光化學 公司) : 包括 (A) 酚型單偶氮染料: 反應紅 198 (RR198, $\left.\lambda_{\max }=522 \mathrm{~nm}\right)$; 蒜酚型雙偶氮染料: 反應黑 $5(\mathrm{RBk} 5$, $\left.\lambda_{\max }=600 \mathrm{~nm}\right)$ 、反應紅141 (RR141, $\left.\lambda_{\max }=544 \mathrm{~nm}\right)$ 、反應藍 $160\left(\mathrm{RBu} 160, \lambda_{\max }=616 \mathrm{~nm}\right)$ 、反應藍171 (RBu171, $\lambda_{\max }=609$ $\mathrm{nm})$ 、反應綠 $19\left(\mathrm{RG} 19, \lambda_{\max }=631 \mathrm{~nm}\right)$ (圖1) (B) 非芸酚型偶 氮染料: 反應黃84 (RY84, $\left.\lambda_{\max }=411 \mathrm{~nm}\right)$ 、直接黃86 (DY86, $\lambda_{\max }=393 \mathrm{~nm}$ ) (圖2), WLP72所用之培養基為Marine Broth 2216 (MB) 其餘為Luria-Bertani (LB)。
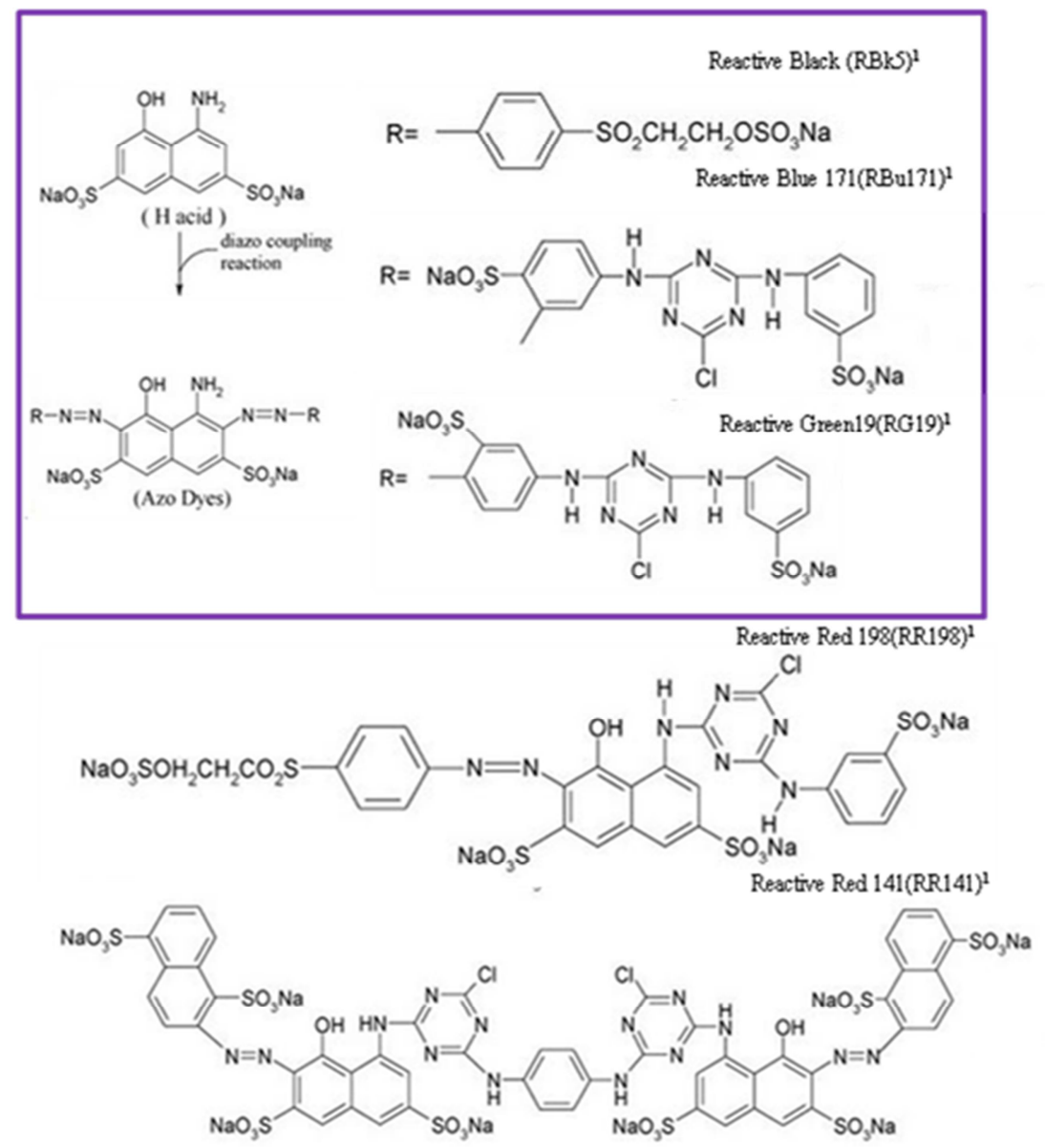

圖1 酚型染料 $[7]$ 。 
<smiles>[R]=C=Cc1ccc2c(S(=O)(=O)O)cc(C)cc2c1S(=O)(=O)[O-]</smiles><smiles>[R]N=Nc1ccc(Nc2nc(NCCO)nc(Nc3ccc(N=N[R])c(C)c3)n2)cc1C</smiles>

Reactive Yellow 84(RY84)<smiles>[R]N=Nc1cc(OC)c(Nc2nc(Cl)nc(Nc3ccc(/C=C\c4ccc(Nc5nc(Cl)nc(Nc6cc(C)c(N=N[R])cc6[S+]([O])([O])[O-])n5)cc4OC)c(S(C)(=O)=O)c3)n2)cc1C</smiles>

圖2 非酚型染料 $[7]$ 。

\section{2. 染料脫色}

首先取凍管菌株 $1 \%(\mathrm{v} / \mathrm{v})$ 接種於新鮮 $\mathrm{LB}$ 基質中 $50 \mathrm{~m} 1$ 中以 $30^{\circ} \mathrm{C} 、 125 \mathrm{rpm}$ 下進行前培養 12 小時進行活化, 再取 前培飬液 $1 \%(\mathrm{v} / \mathrm{v})$ 於含有 $200 \mathrm{mg} \mathrm{L}^{-1}$ 染料之相同新鮮LB培 養基中 $50 \mathrm{ml}$ 進行搖瓶培養, 後搖瓶 6 小時 $\left(30^{\circ} \mathrm{C} 、 125 \mathrm{rpm}\right)$, 開始靜置, 並以個時間取出測量其菌種濃度與染料濃度 (以分光光度計測得), 菌體濃度測 $0 \mathrm{D}_{600 \mathrm{~m}}$; 染料濃度測其 最大吸收峰 $\lambda_{\max }$ 。

\section{3. 分析方法}

經由分光度計, 染料 (染料之最大吸收峰 $\lambda_{\max }$ ) 和菌體 $\left(\lambda_{\text {max }}=600\right.$ m $)$ 。濃度之測量可以下列方式決定 $[6]$ :

（a）假設未經離心之原含菌液樣品之測量值為:

$$
O D_{600 \mathrm{~nm}}^{X+D Y E}=O D_{600 \mathrm{~nm}}^{D Y E}+O D_{600 \mathrm{~nm}}^{X}
$$

（b）經離心 (12000 rpm $\approx 14940 X g$ ) 取上層液（假設含染 料) 所測得值為

$$
O D_{600 \mathrm{~nm}}^{\mathrm{sup}}=O D_{600 \mathrm{~nm}}^{D Y E}
$$

（c）經離心 (12000 rpm $\approx 14940 X g$ ) 取上層液（假設含染 料) 所測得值為

$$
O D_{\lambda \max }^{\mathrm{sup}}=O D_{\lambda \max }^{D Y E}
$$

將此三個方程式聯立即可算出DYE濃度 $O D_{\lambda \text { max }}^{D Y E}$ 和 $O D_{600 n m}^{X}$, 再取適當的稀釋倍數於線性範圍內 $(0 \mathrm{D}<0.6)$, 最後才由檢量線計算實際濃度。
再以每單位菌體濃度計算比生長速率 (specific growth rate, SGR) 和比脫色速率 (specific decolorization rate, SDR), 其計算和定義如下:

Logistic equation:

$$
\frac{d \ln X}{d t}=\mu\left(1-\frac{X}{X_{0}}\right)
$$

Specific growth rate $\left(h^{-1}\right)$ :

$$
S G R=\frac{d \ln X}{d t} \cong \frac{1}{X} \frac{\Delta(X)}{\Delta t}
$$

Specific decolorization rate $\left(\mathrm{mg} \mathrm{L}^{-1} \mathrm{~h}^{-1} \mathrm{ODU}^{-1}\right)$

$$
S D R=-\frac{1}{X} \frac{d[D Y E]}{d t}
$$

$X$ 為菌體濃度、 $X_{0}$ 為起始菌體濃度、 $\mu$ 為生長速率 $[D Y E]$ 濃度 $\left(\mathrm{mg} \mathrm{L}^{-1}\right) 、 \mathrm{t}$ 為時間 $(\mathrm{h})$

\section{3. 結果與討論}

\section{1. 染料結構效應影響脫色效率}

依染料結構影響脫色速率之主要因素, 可分為下列三 種 [7]：（一) 偶氮腙互變異構(圖3) [19];（二）電子效應(圖 4 ); 和 (三) 立體阻礙效應 (圖1)。

先前研究 [7]指出鄰位菜酚類偶氮染料較非䒺酚類偶 氮染料更易進行微生物脫色, 主要原因是銼基位於䒺酚環 的第二個碳上, 會發生偶氮 - 腙互變異構 (如: $-\mathrm{N}=\mathrm{N}-\leftrightarrow$ $=\mathrm{N}-\mathrm{NH}$ ) (圖3), 因此為推電子基之羥基 $(-\mathrm{OH})$ 經由結構異 
構化後將轉為成拉電子基之羰基 $(\mathrm{C}=0)$, 由於羰基是具高 陰電性，會降低偶氮鍵的電子密度，因而有利於偶氮染料 進行還原脫色外，拉電子基之羰基也可以穩定在還原時所 產生其負電荷 (圖4)。此鄰位萗酚類偶氮染料較非䒺酚類 偶氮染料更易進行微生物脫色之現象在A1-Amrani [14]等 人亦曾提及。

WLP72對多種染料進行脫色時，其中對RBu160脫色速 率為最快外, 其他偶氮染料的比脫色速率 (SDR) 排序如

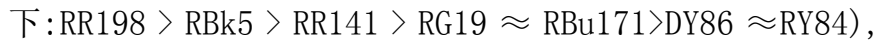
此順序大致與NIU01 [7] (表1) 對菜酚類染料有著平行之次 序 (即是RR198>RBk5>RR141>RG19 $\approx \mathrm{RBu} 171$ ), 所以 發現WLP72對不同偶氮染料的結構效應大致如同NIU01 [7] 符合上述之討論說明。

拉電子基在偶氮鍵的對位處和鄰位處時會因為有共 振效應和感應效應而降低偶氮雙鍵的電子密度, 促使偶氮 雙鍵的pi鍵在進行還原脫色時較容易被分解打斷 (圖4)。 並從不同染料脫色結果發現拉電子基在不同位置時會對 脫色效率有顯著影響。亦就是拉電子基在偶氮鍵的對位處 會比在鄰位處更容易脫色。例如在對位處者 如:RR198 (53.7)、RBk5 (48.8) 即具有較高比脫色速率 (SDR); 而在鄰位處者如：RR141 (36. 3)、RBu171(13.7) 之SDR 則較低, 其因即是在鄰位處立體阻礙大於在對位 處。

RR198 比RBk5 快其原因為RR198為單偶氮染料，RBk5 為雙偶氮染料, RR198只要打斷一個偶氮鍵即可達去色效 果。至於RBk5 比RR141快之主因是為其立體阻礙較小 (圖1)。 另外, RR141比RBu171和RG19快，主要原因是RBu171和RG19 分別在偶氮鍵的對位和間位處有推電子基 (胺基- $\mathrm{NH}-$ ), 會 使偶氮雙鍵電子雲密度上升，使其偶氮雙鍵更穩定，比較 不容易被還原分解打斷[7]。<smiles>Nc1c(N=Nc2ccc(S(=O)(=O)OCCS(=O)(=O)O[Na])cc2)c([S+](=O)(=O)O[Na])cc2cc(S(=O)(=O)O[Na])c(N=Nc3ccc(S(=O)(=O)O[Na])cc3)c(O)c12</smiles><smiles>Nc1c(N=Nc2ccc(S(=O)(=O)OCCOS(=O)(=O)O[Na])cc2)c(S(=O)(=O)O[Na])cc2c1C(=O)C(=NN([TlH])c1ccc(S(=O)(=O)O[Na])cc1)C(S(=O)(=O)O[Na])=C2</smiles>

圖3 偶氮腙互變異構圖 [7]。

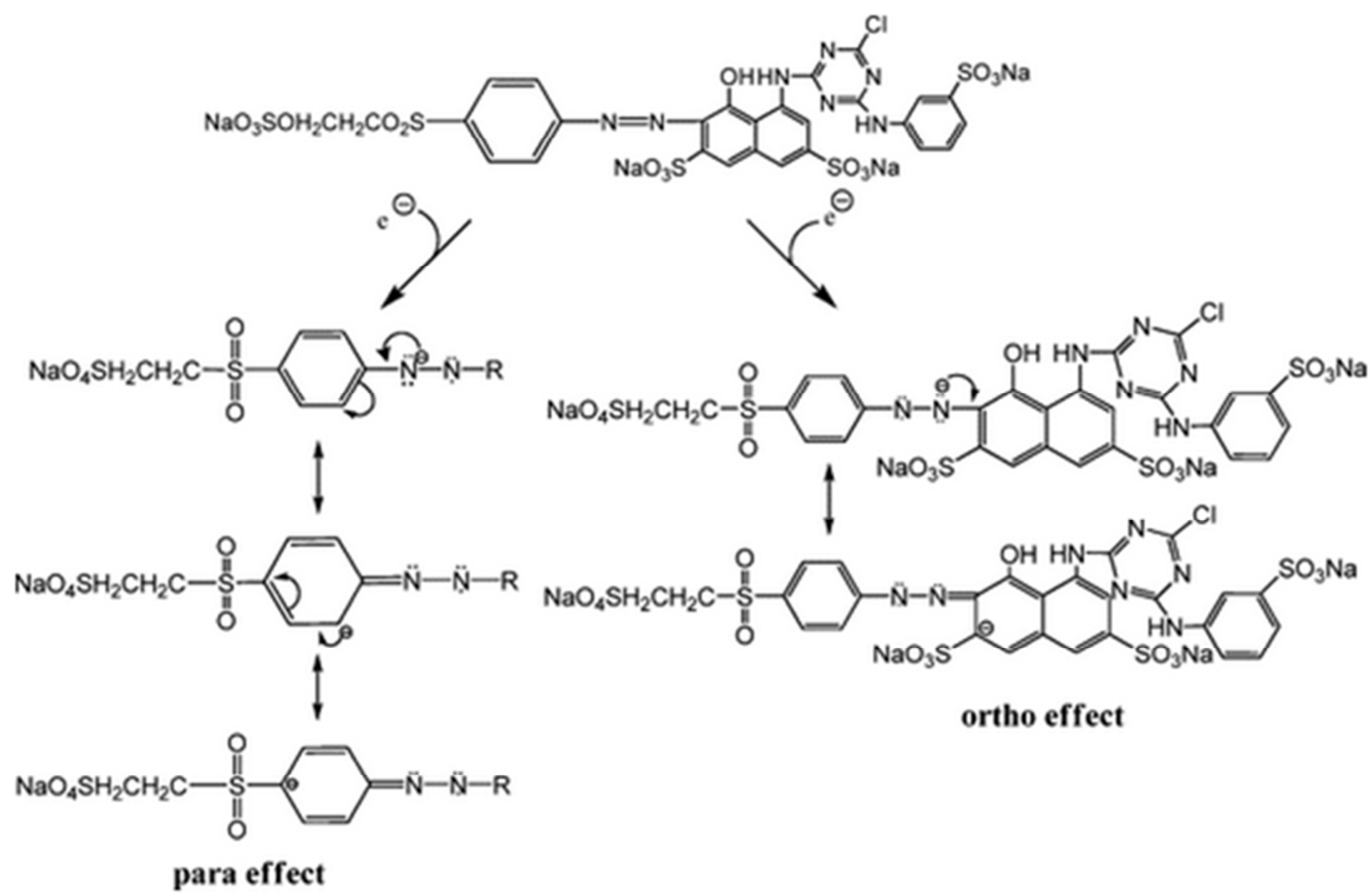

圖4 染料結構 - 電子效應 $[7]$ 。 
表1 WLP72與NIU01對不同偶氮染料之脫色效率比較。

\begin{tabular}{lll}
\hline \multirow{2}{*}{ 染料類型 } & \multicolumn{2}{l}{ Decolorization rate $\left(\boldsymbol{\mu M ~ \mathrm { h } ^ { - 1 }} \mathbf{O D U}^{-1}\right)$} \\
\cline { 2 - 3 } & WLP72 & NIU01 [7, 8] \\
\hline Reactive Blue 160 & $62 \pm 2$ & 16.7 \\
Reactive Red 198 & $57 \pm 3$ & 264.2 \\
Reactive Black 5 & $48 \pm 1$ & 81.6 \\
Reactive Red 141 & $36 \pm 1$ & 37.5 \\
Reactive Green 19 & $17 \pm 4$ & 25.4 \\
Reactive Blue 171 & $17 \pm 3$ & 29.0 \\
Direct Yellow 86 & $13 \pm 1$ & 20.7 \\
Reactive Yellow 84 & $12 \pm 1$ & 16.4 \\
\hline
\end{tabular}

\section{2. 希瓦氏菌WLP72對不同偶氮染料之脫色能力}

以希瓦氏菌WLP72對各種染料進行脫色，可從圖5及表 2看出, WLP72對於各種染料在一天内皆能有效降解脫色至 71. $4 \%$ 以上。且從表 2 可看出, 希瓦氏菌WLP72之脫色能力 比起其他脫色菌株還來的優異許多。Shewanella sp. WLP72, 具有奈米導線可增強電子之傳導, 由於生物染料 脫色為還原機制, 故WLP72具有良好之脫色能力。希瓦氏 菌不僅僅可降解偶氮染, 再由Chen[9]和Cao[12]等人更發 現亦可以降解非偶氮染料。Cao[12] 等人以 Shewanella oneidensis MR-1 對 methyl orange (M0) 和 naphthol green B (NGB) 之混合液具有很強厥氧脫色能力, 然而其 對M0具有更強脫色能力, 且M0會抑制NGB還原脫色。其也 提及添加核黃素 (riboflavin) 作為電子梭會加速脫色速 率，推測Shewanella oneidensis MR-1之脫色機制非僅只 涉還原酵素, 另會產生電流加強脫色效率. 依本研究結果 亦發現, 希瓦士菌WLP72對各種染料皆有不錯的降解脫色 能力, 即具有對各種染料較廣泛之脫色能力。

表2 不同的本土脫色菌對偶氮染料 (RBk5和RBu160) 之脫色活性 (SDR) 比 較表。

\begin{tabular}{|c|c|c|c|c|}
\hline Strains & 編號 & RBk5 & RBu160 & 篩選方式及條件 \\
\hline \multirow{5}{*}{$\begin{array}{l}\text { Acinetobacter } \\
\text { johnsonii } \\
\text { Acinetobacter } \\
\text { juni i } \\
\text { Aeromonas } \\
\text { hydrophila }\end{array}$} & NIUx72 & 5.97 & $5.74 \mathrm{~b}$ & RBu198、RY84 /LB \\
\hline & NIUY8 & 7. 86 & 3. $51 \mathrm{c}$ & MFC (MM) \\
\hline & NIU01 & 81. $6 \mathrm{a}$ & $16.7 \mathrm{~b}$ & RR141/LB \\
\hline & NIUx73 & 1.76 & 9. $46 \mathrm{~b}$ & RBu198、RY84 /LB \\
\hline & YT11 & 65.89 & 11. $76 \mathrm{~b}$ & RBu160、RBk5/LB \\
\hline Aeromonas & NIUP9 & 2. 66 & 4. $90 \mathrm{c}$ & $\operatorname{MFC}(\mathrm{MM})$ \\
\hline $\begin{array}{l}\text { Enterobacter } \\
\text { cancerogenus }\end{array}$ & BYm30 & 1. 62 & $.51 \mathrm{~b}$ & RBu160、RBk5/LB \\
\hline Exiguobacterium & K4 & $26.4 \mathrm{~d}$ & 7. $68 \mathrm{~d}$ & $\mathrm{NaCl} / \mathrm{LB}$ \\
\hline $\begin{array}{l}\text { Klebsiella } \\
\text { pneumoniae }\end{array}$ & ZMd31 & 2. 02 & 2. $71 \mathrm{~b}$ & RBu160、D039/LB \\
\hline $\begin{array}{l}\text { Klebsiella } \\
\text { variicola }\end{array}$ & P11 & $1.2 \mathrm{~d}$ & 1. $17 \mathrm{~d}$ & Phenol \\
\hline Proteus hauseri & ZMd44 & 3. $43 \mathrm{~b}$ & 11. $16 \mathrm{~b}$ & RBu160、D039/LB \\
\hline Shewanella sp. & WLP72 & 48.0 & 62.0 & $\mathrm{RBu} 160 / \mathrm{MB}$ \\
\hline $\begin{array}{l}\text { Staphylococcus } \\
\text { gallinarum }\end{array}$ & K1 & 14. $11 \mathrm{~d}$ & $2.9 \mathrm{~d}$ & $\mathrm{NaCl} / \mathrm{LB}$ \\
\hline
\end{tabular}

$\mathrm{a}:[7], \mathrm{b}:[8], \mathrm{c}:[10], \mathrm{d}:[11]$.

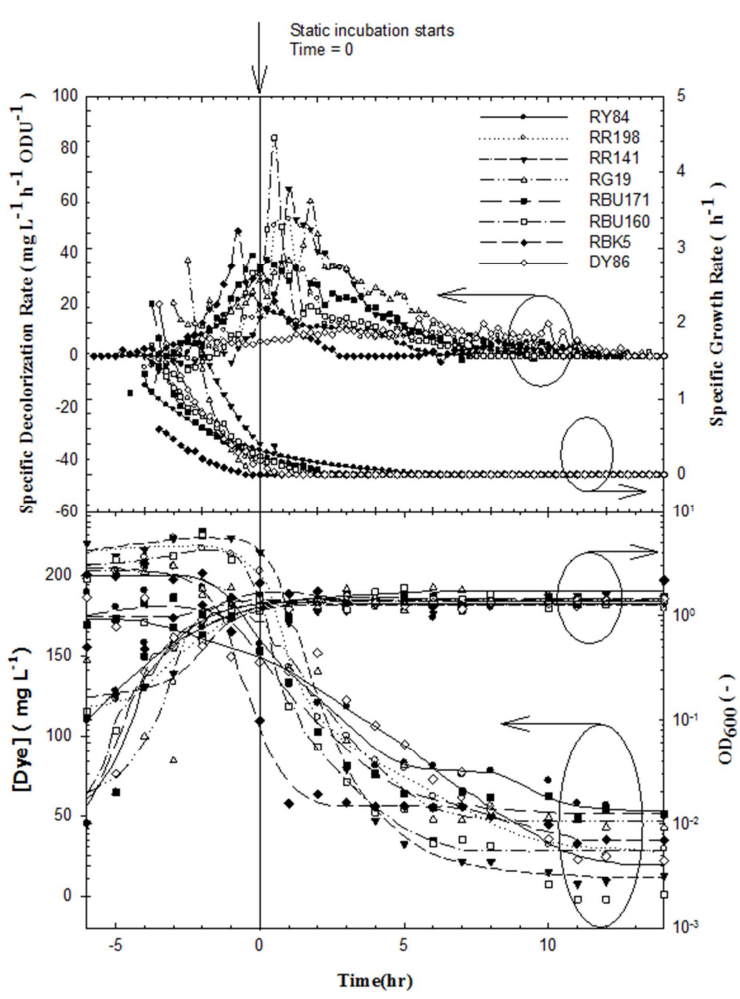

圖5 以WLP72對各種染料進行生長及脫色。

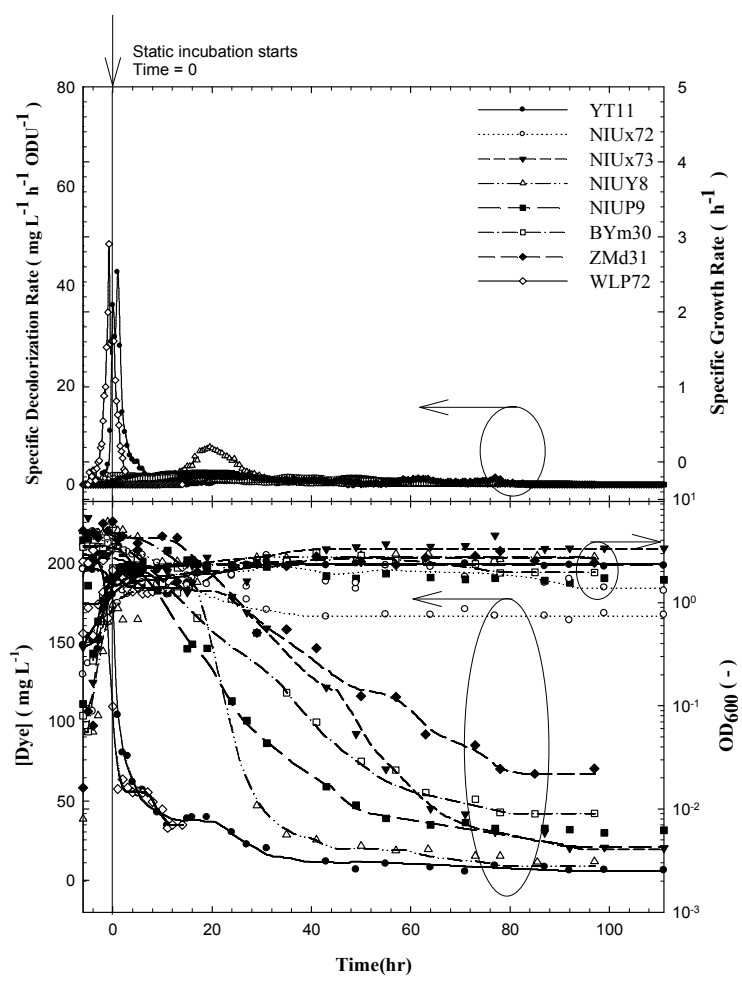

圖6 不同的本土脫色菌對RBk5之生長及脫色曲線。

\section{3. 比較不同脫色菌株之脫色能力}

為針對各種脫色菌株之脫色活性做量化比較以選定 $R B k 5$ 和RBu160為目標染料, 對RBk5染料 (圖6、表2)之比脫 色速率 $\operatorname{SDR}\left(\mu \mathrm{M} \mathrm{h}^{-1} 0 \mathrm{OU}^{-1}\right)$, 其大小次序如下列: 
$\operatorname{NIU01}(81.6)>\mathrm{YT} 11(65.89)>\operatorname{WLP} 72(48.0)>\mathrm{K} 4(26.4)>$ $\mathrm{K} 1(14.11)>\operatorname{NIUY8}(7.86)>\operatorname{NIUx} 72(5.97)>\mathrm{ZMd} 44(3.43)>$ $\operatorname{NIUP9}(2.66) \approx \operatorname{ZMd} 31(2.02) \approx \operatorname{BYm} 30(1.62) \approx \operatorname{NIUx} 73$ $(1.76) \approx \mathrm{P} 11(1.2)$. 另外, 對 RBu160脫色之 $\operatorname{SDR}\left(\mu \mathrm{M} \mathrm{h}^{-1}\right.$ $\left.\mathrm{ODU}^{-1}\right)$ 如下所示:WLP72 (62.0) > NIU01 (16.7)>YT11 (11. 76) $\approx$ ZMd44 (11. 16)>NIUx73 (9.46)>K4(7.68)> NIUx72 $(5.74)>\operatorname{NIUP9}(4.90) \approx \operatorname{NIUY8}(3.51) \approx \operatorname{K1}(2.9) \approx \mathrm{ZMd} 31$ $(2.71) \approx \operatorname{BYm} 30(2.51) \approx \mathrm{P} 11(1.17)$ 。除了NIUx73和ZMd44 例外, 其他各脫色菌株對RBk5和RBu160脫色活性能力大致 是平行。在Peres [15]和Hsueh [16] 等人即發現不同菌屬在 還原4-nitro-benzoic acid(4-NBA) 時就因為具有不同之 生物還原途徑以致會產生不同代謝物。所以此些不同菌屬 脫色菌株或源自篩選條件不同, 是以對偶氮染料脫色具有 不同脫色能力, 其中以同為Aeromonas hydrophila 的 NIU01, YT11和 Shewanella sp. WLP72此三株菌對RBk5 和 $\mathrm{RBu} 160$ 均具有不錯脫色能力。

\section{4. 篩選條件}

穊選條件是會對脫色菌之脫色能力有影響 [17], 對 RBu160 之脫色效率有優於 RBk5 之菌株, 除了 NIUx73 和 NIUP9者, 即是有以RBu160為篩選條件之菌株者, 例如 ZMd31、ZMd44、WLP72，且其中希瓦氏菌WLP72則僅是以 RBu160為篩選條件, 因此對RBu160之脫色能力尤其具有特 別強化之優勢效果。然而BYm30和YT11則也有用RBk5為篩 選條件, 故二者對RBu160脫色效果並未明顯優於RBk5。

\section{4. 1. 以非偶氮染料穊選之菌株}

若以 RBu198之非偶氮染料作為篩選條件之菌株如 NIUx72和NIUx73, 對於偶氮染料之脫色活性則較差, 可能 由於以非偶氮染料篩出的菌株是以未具有顯著的 $\mathrm{azo}^{-}$ reductase活性 [18], 所以對偶氮染料之脫色活性都不高, 導致脫色能力不佳。

\section{4. 2. 貧瘦培養基}

從表 2 指出, 若是以太貧瘦的培養基作為碳源以較貧 㾉之培養基 (MM) 所篩出之菌株, 為MFC為篩選條件所得之 產電菌, 且發現若是以此種當作培養基篩出的菌例, 如 NIUY8 和NIUP9之脫色能力幾乎都是最差的。

\section{4. 3. 不同篩選條件之同屬脫色菌}

同為Aeromonas屬之NIU01、YT11、NIUx73 和NIUP9則 具有不同脫色能力: NIU01>YT11>NIUx73>NIUP9。 其中NIU01和YT11和NIUx73為同屬同種, 但篩選條件不同。 由於NIU01是由毒性較高且較不易脫色的偶氮染料RR141 優勢篩選出來 [13], 故其脫色能力極為優異。而YT11則是 由偶氮染料RBk5和RBu160兩種脫色活性所篩選, 是以對偶 氮染料具有不錯之脫色能力。NIUx73則是由非偶氮染料 RBu198與偶氮染料RY84篩選, 通常以非偶氮染料篩選之菌 株, 對偶氮染料脫色能力較差。而NIUP9則是glucose /acetate做為碳源以較貧痊之培養基 (MM), 以MFC為篩選 條件所得之崖電菌, 且發現若是以此種當作培養基篩出的 菌, 脫色能力幾乎都是較差的例如NIUY8。

\section{4. 4. 耐鹽菌株和耐酚菌株}

以耐鹽菌 $\mathrm{K} 4$ 而言笁選階段並無添加染料, 雖是僅以高 鹽度進行篩選。但是LB仍可促進K4對偶氮染料脫色效果 [11] 和降解硝基芳香化合物 [16], 可能是LB內成分可促進 K4的還原酵素活性有關。另外, 耐酚菌株P11是以酚為篩 選條件 [11], 所以其對偶氮染料脫色能力在所有脫色菌中 是最差。

\section{4. 結論}

WLP72對萘酚型染料脫色活性次序與NIU01相似, 染料 結構效應對WLP72脫色活性的影響類似NIU01。本研究發現 穊選條件對菌株有明顯影響脫色效能, 以RBu160為籂選條 件之WLP72對RBu160之脫色效率甚至比易脫色的䒺酚型染 料快, 且高於其他脫色菌。但若以極為貧痊之培養基或非 偶氮染料作為節選條件, 所分離純化的菌株, 對偶氮染料 脫色能力都較差。

\section{致谢}

感謝科技部計畫 (MOST 103-2221-E-197-021) 和 （MOST 104-2221-E-197-030）之經費補助。

\section{参考文献}

[1] Frank P, V. d. Z. , Cervantes, F. J., Impact and application of electron shuttles on the redox (bio) transformation of contaminants: A review $[\mathrm{J}]$. Biotechnol. Adv. V. 27, 2009, p 254-277.

[2] Stolz, A., Basic and applied aspects in the microbial degradation of azo dyes [J]. Appl. Microbiol. Biot. V. 56, 2001, p 68-80.

[3] Paździor, K., Klepacz-Smółka, A., Ledakowicz, S., Sójka-Ledakowicz, J., Mrozińska, Z., Ży łła, R., "Integration of nanofiltration and biological degradation of textile wastewater containing azo dye [J]. Chemosphere. V. 75, 2009, p 250-255.

[4] Khalid, A., Arshad, M., Crowley, D., Decolorization of azo dyes by Shewanella sp. under saline conditions [J]. Appl. Microbiol. Biot. V. 79, 2008, p 1053-1059.

[5] Fernando, E., Keshavarz, T., Kyazze, G., Complete degradation of the azo dye Acid Orange-7 and bioelectricity generation in an integrated microbial fuel cell, aerobic two-stage bioreactor system in continuous flow mode at ambient temperature $[\mathrm{J}]$. Bioresource Technol. V. 156, 2014, p 155-62.

[6] Chen, B. Y., Understanding decolorization characteristics of reactive azo dyes by Pseudomonas luteola: toxicity and kinetics [J]. Process. Biochem. V. 38, 2002, p 437-446. 
[7] Hsueh, C. C., Chen, B. Y., Yen, C. Y., Understanding effects of chemical structure on azo dye decolorization characteristics by Aeromonas hydrophila [J]. J. Hazard. Mater. V. 167, 2009, p 995-1001.

[8] Zhang, M. M. , Chen, W. M. , Chen, B. Y., Chang, C. T. , Hsueh, C. C., Ding, Y., Lin, K. L., Xu, H., "Comparative study on characteristics of azo dye decolorization by indigenous decolorizers [J]. Bioresource. Technol. V. 101, 2010, p $2651-2656$.

[9] Chen, C. H., Chang, C. F., Ho, C. H., Tsai, T. L., Liu, S. M. , Biodegradation of crystal violet by a Shewanella sp. NTOU1 [J]. Chemosphere. V. 72, 2008, p 1712-1720.

[10] Han, J. L., Liu, Y., Chang, C. T., Chen, B. Y., Chen, W. M., Xu, H. Z., Exploring characteristics of bioelectricity generation and dye decolorization of mixed and pure bacterial cultures from wine-bearing wastewater treatment $[\mathrm{J}]$. Biodegradation. V. 22, 2011, p $321-333$.

[11] Chen, B. Y., Hsueh, C. C., Chen, W. M. , Li W. D., Exploring decolorization and halotolerance characteristics by indigenous acclimatized bacteria: Chemical structure of azo dyes and dose-response assessment [J]. J. Taiwan. Inst. Chem. Eng. V. 42, 2011, p $816-825$.

[12] Cao, D. -M. , Xiao, X. , Wu, Y.-M. , Ma, X. -B. , Wang, M. -N. , Wu, Y. - Y., Du, D. -L., Role of electricity production in the anaerobic decolorization of dye mixture by exoelectrogenic bacterium Shewanella oneidensis MR-1 [J]. Bioresource Technol.V. 136, 2013, p 176-181.
[13] Chen, B. Y., Chen, W. M., Wu F. W. , Chen, P. K. , Yen, C. Y., Revealing azo-dye decolorization of indigenous Aeromonas hydrophila from fountain spring in Northeast Taiwan [J]. J. Chin. Inst. Eng. V. 39, 2008, p 495-501.

[14] A1-Amrani, W. A., Lim, P. -E., Seng, C. -E., Ngah, W. S., W. , Factors affecting bio-decolorization of azo dyes and COD removal in anoxic-aerobic REACT operated sequencing batch reactor $[\mathrm{J}]$. J. Taiwan Inst. Chem. Eng. 45, 2014, p 609-616.

[15] Peres, C. M., Russ, R., Lenke, H., Agathos, S. N. , Biodegradation of 4-nitrobenzoate, 4-aminobenzoate and their mixtures: new strains, unusual metabolites and insights into pathway regulation $[\mathrm{J}]$. FEMS Microbiol. Ecol. 2001, 37, 151-159.

[16] Hsueh, C. -C. , You, L. -P., Li, J.-Y., Chen, C. -T. , Wu, C. - C. , Chen, B. - Y. , Feasibility study of reduction of nitroaromatic compounds using indigenous azo dye-decolorizers [J]. J. Taiwan Inst. Chem. Eng. , 2016, in press.

[17] Sridhar Rao, P. N., Bacterial culture media [R]. http://www. microrao. com/micronotes/culture_media. p df, 2012.

[18] Singh, S. N., Microbial Degradation of Synthetic Dyes in Wastewaters [C]. Allan, R., Förstner, U., Salomons, W. Ed., Environmental Science and Engineering, Environmental Science, 2015.

[19] Pearcea, C. I., Lloydb, J. R., Guthriea, J. T., The removal of colour from textile wastewater using whole bacterial cells: a review $[\mathrm{J}]$. Dyes and Pigments 58 (2003) $179-196$. 